regelmäßig vorhanden, daß sie nur als Stadien einer echten planktischen Entwicklung angesprochen werden konnten, wie sie u. W. für die Angehörigen der Familie Tubulariidae bislang nicht bekannt ist.

Bei Untersuchungen über die Fortpflanzung und Entwicklung von Margelopsis haeckeli Hartlaub erhielt WerNER am 22. 7. und 18. 8. 1953 aus dem Plankton des Nordsylter Wattenmeeres zufällig je eine lebende Actinula des Stadiums 2, die in Kultur genommen werden konnte. Die Actinulae hefteten sich jeweils bereits nach 2 Tagen fest und entwickelten sich zu kleinen Polypen, die nach ihrer Morphologie nur die Polypen von Ectopleura dumortieri sein konnten. Der endgültige Beweis für die Artzugehörigkeit ließ sich bald darauf führen, als es am 20. 8. 1953 gelang, von geschlechtsreifen EctopleuraMedusen befruchtete Eier zu gewinnen, die sich in den Kulturgläsern weiterentwickelten. Daher konnten ihre Furchung und die weitere Entwicklung über das Stadium 1 bis zum Stadium 2 der ansatzreifen Actinula direkt beobachtet werden. Mehrere Actinulae setzten sich auf dem Boden der Kulturgläser fest. Die sich aus ihnen entwickelnden Polypen konnten längere Zeit weiter gezüchtet werden, bis sie Medusen bildeten; damit war der Entwicklungszyclus vollständig.

Entsprechend dem Gang der Beobachtung und Untersuchung wird der eine von uns über die Verbreitung und das zeitliche Auftreten der Entwicklungsstadien im Plankton der südlichen Nordsee berichten und dabei auch die Medusengeneration einbeziehen, der andere über die Entwicklung des Polypen aus dem befruchteten Ei.

\title{
I. Die Entwicklung des Polypen von Ectopleura dumortieri van Beneden
}

\author{
Von Bernhard Werner
}

(Mit 8 Abbildungen und 1 Tabelle im Text)

\section{A. Einleitung}

Bei der Neubearbeitung der Systematik der Tubulariiden stellt Kramp (1949) in Anlehnung an ältere Autoren (z. B. KüHN 1913) die Superfamilie der Tubulariformes auf und unterscheidet: A. die Tubularia-Linie mit den Familien Tricyclusidae, Margelopsidae (Gattungen Margelopsis, Pelagohydra, Climacodon), und Tubulariidae (Gattungen Ectopleura, Hybocodon, Tubularia); B. die Corymorpha-Linie mit den Familien Asyncorynidae, Boreohydridae, Corymorphidae (Gattungen Heteractis-Euphysa, Gymnogonus, Corymorpha-Steenstrupia) und Branchiocerianthidae.

Dieses System stützt sich im wesentlichen auf die vergleichende morphologische Untersuchung der Hydroidengeneration. Wie die Zusammenfassung der Gattungen Ectopleura, Hybocodon und Tubularia zur Familie Tubulariidae zeigt, ist das Vorkommen von freischwimmenden Medusen bzw. von sessilen Gonophoren kein Merkmal von systematischer Bedeutung. Bemer- 
kenswert erscheint, daß der Polyp bei vielen Formen der beiden großen Gruppen solit är lebt. Darüber hinaus nehmen die Margelopsidae eine Sonderstellung ein, weil bei ihnen der Polyp ein pelagisches Dasein führt, während er bei den anderen Formen in der üblichen Weise angeheftet ist oder sich mit dem basalen Teil des Hydrocaulus in den Boden eingräbt.

Soweit die Entwicklungsgeschichte der Hydroidengeneration bekannt ist, scheint das wesentliche gemeinsame Merkmal das Fehlen einer freischwimmenden Planulalarve zu sein. Innerhalb der T ubularia-Linie entwickelt sich das Ei zu dem larvalen Stadium der Actinula, die zum Bodenleben übergeht bzw. in der Familie Margelopsidae das planktische Leben beginnt. Dieser Entwicklungsmodus ist seit langem bekannt für die am eingehendsten untersuchte Gattung Tubularia, die sessile Gonophoren hat, bei der dementsprechend die direkte Entwicklung weniger auffällig erscheint. Aber auch bei den Formen, bei denen ein typischer Generationswechsel ausgebildet ist, kommt der gleiche Entwicklungsgang vor. Das ist bisher für die Gattungen Hybocodon und Margelopsis beschrieben, bei denen die Entwicklung des Eies zur Actinula am Manubrium der Meduse stattfindet. Für Margelopsis ist noch hervorzuheben, daß dieser Entwicklungsgang nur für die zuerst gebildeten kleineren Subitaneier zutrifft (WERNER 1954, 1955 a, b, s. u. S. 245 f.).

Wie die hier mitgeteilten Beobachtungen zeigen werden, schließt sich Ectopleura durch die direkte Entwicklung vom Ei zur Actinula unmittelbar an, mit dem Unterschied, daß sich das befruchtete Ei vom Manubrium ablöst und die Entwicklung zur ansatzreifen Actinula pelagisch durchläuft.

Für die Entwicklungsgeschichte der Hydroidengeneration innerhalb der Corymorpha-Linie sei hier nur auf den Entwicklungsmodus von Corymorpha hingewiesen. Wie Torrey (1907) für C. palma und Rees (1937) für C. nutans gezeigt haben, löst sich das befruchtete Ei vom Manubrium los und sinkt zu Boden, wo es sich anheftet. Nach wenigen Tagen schlüpft ein zunächst wenig differenzierter kleiner Polyp aus, der sofort zum Bodenleben übergeht und sich kontinuierlich zum definitiven Polypen weiterentwickelt. Ein ausgesprochenes Actinulastadium fehlt hier also. Wie weit die Entwicklungsgeschichte bei den übrigen Familien der Corymorpha-Linie bekannt ist oder sich in diesen Entwicklungstypus einfügt, kann hier nicht geprüft werden; erwähnt sei nur, daß das Fehlen der Actinula bei ihnen allgemein zuzutreffen scheint (Kramp 1949).

\section{B. Die Entwicklung des Polypen}

Der Polyp von Ectopleura wird oft an Stöckchen von anderen Hydrozoen angeheftet gefunden und lebt meist solitär; nur gelegentlich wird ein einzelner Seitenzweig beobachtet. Der zarte Hydrocaulus, der nach MAYER (1910) etwa $25 \mathrm{~mm}$ lang wird, nach eigenen Beobachtungen aber eine Länge von $10 \mathrm{~cm}$ erreichen kann, hat eine rötliche Färbung und eine feine Längsstreifung. Das Periderm ist gelblich gefärbt und hat in der Nähe des Hydranthen eine schwache Ringelung. Am Polypenkopf befinden sich zwei Tentakelkränze; der Mundpol trägt einen Kranz von 15-25 kürzeren Oraltentakeln, der aborale Pol einen Kranz von 20-30 längeren Tentakeln. Dicht über diesen Aboraltentakeln liegt die Knospungszone für die Medusen. Sie werden gruppenweise an kurzen, wenig verzweigten Gonophorenträgern gebildet. 
Die wichtigsten Kennzeichen der Meduse sind das Vorhandensein der 4 Paare von Nesselzellstreifen auf der Exumbrella, sowie die Ausbildung der Tentakel. An jedem der 4 kräftigen Tentakelbulben setzt je 1 vollentwickelter Tentakel an, der an der Dorsalseite getrennte spangenartige Anhäufungen von Nesselzellen trägt und sich bei der Kontraktion spiralig einrollt.

Die Medusen sind getrenntgeschlechtig. Die Gonade liegt im Ektoderm des Manubriums und umgibt es röhrenförmig. Das Keimepithel ist beim Weibchen meist sehr dünn und verdickt sich nur an den Stellen, an denen sich die Eier entwickeln. Dabei kommen jeweils immer nur wenige Eizellen gleichzeitig zur Entwicklung, meist nur zwei. Wie auch aus der Entwicklung der Eizelle bei den anderen Tubulariiden bekannt ist, nimmt sie während ihres Wachstums Material von anderen Oogonien auf, die als Nährzellen dienen (s. u. S. 247). Die Kerne dieser Nährzellen, die der Auflösung verhältnismäßig lange wiederstehen, bleiben als sog. „Pseudozellen“ längere Zeit erhalten und sind bei Ectopleura an dem reifen, eben abgelegten $\mathrm{Ei}$, wie auch an den jüngeren Furchungsstadien von außen in größerer Zahl deutlich zu erkennen (Abb. 1).

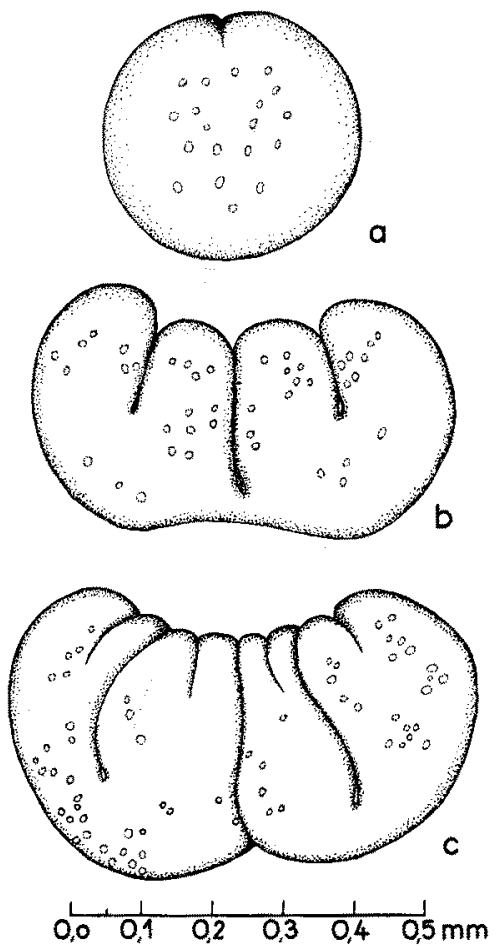

Abb. 1 a-c. Ectopleura dumortieri, Stadien der Eifurchung bis zum beginnenden 8-Zellstadium. Abbéscher Zeichenapp. v, Leitz.

Da nur wenige reife Weibchen zur Verfügung standen, war die Zahl der von ihnen abgelegten Eier gering. Doch reichen sie zur Beschreibung der Besonderheiten der Entwicklung aus.

Nach dem Austritt aus der Gonade lösen sich die Eier sofort vom Manubrium los. Ob sie bereits während des Austrittes aus dem Keimepithel oder erst nach der Ablösung besamt werden, konnte nicht beobachtet werden. Die 
relativ großen Eier haben eine gelblich-weiße Farbe und sind ziemlich transparent. Der Durchmesser betrug bei zwei Eiern 0,312 und 0,325 mm. Ihr spezifisches Gewicht ist allem Anschein nach sehr gering. In den Kulturschalen lagen sie zwar auf dem Boden, doch hefteten sie sich nicht fest und gerieten schon durch die schwachen Konvektionsströmungen ins Treiben, die durch die geringe Erwärmung bei der Untersuchung im durchfallenden Licht einer Monlalampe entstanden. Daraus kann geschlossen werden, daß die Eier im freien Wasser, das ja überdies ständig bewegt ist, pelagisch sind (vgl. ThoRson 1946, p. 421).

Bei der Teilung verlaufen die einzelnen Furchungsschritte mit ziemlicher Geschwindigkeit. Das 8-Zellstadium wurde bei einer Zimmertemperatur von $17-19^{0} \mathrm{C}$ ca. $2^{1 / 2}$ Std. nach der Eiablage erreicht. Die Art der Zellteilung weicht von dem für Anthomedusen üblichen Furchungsmodus stark ab, da die ersten drei Furchen vertikal verlaufen und als sog. "schneidende Furchen "in Erscheinung treten $(A b b .1 \mathrm{a}-\mathrm{c})$. Das 2-Zellstadium nimmt dadurch die sog. "Hantelform" an. Noch ehe die beiden ersten Blastomeren vollständig getrennt sind, setzt bereits der zweite Furchungsschritt ein; das gleiche gilt auch für das 4-Zellstadium.

Die weiteren Zellteilungen wurden nicht beobachtet; 4 Std. nach Furchungsbeginn lag das in Abb. 2 dargestellte langgestreckte Aggregat von größeren und kleineren Zellen vor, bei dem die einzelnen Zellen bei der äußeren Betrachtung nur schwer abzugrenzen waren, und bei dem sich eine bestimmte Ordnung nicht mehr erkennen ließ.

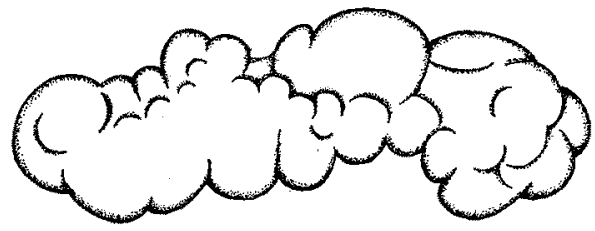

Abb. 2. Ectopleura dumortieri, Furchungsstadium, 4 Std. nach der Eiablage. Vergrößerungsmaßstab wie in Abb. 1. Abb. Zeichenapp. v. Leitz.

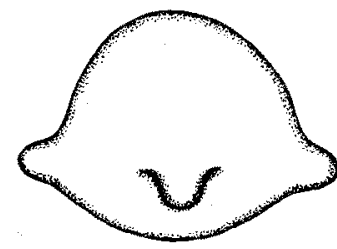

Abb. 3. Ectopleura dumortieri, Entwicklungsstadium mit beginnender Ausbildung der aboralen Tentakel. Freihandzeichnung.

$\mathrm{Daß}$ es sich aber um einen normalen und nicht pathologischen Furchungsablauf gehandelt hat, zeigt die Weiterentwicklung zur beginnenden Actinula, die eine charakteristische Form hat und oben als "Sternchenstadium" bezeichnet wurde. Dieses Stadium der "Proactinula" war 22 Std. nach der Eiablage ausgebildet (Abb. 4 a, b). Bei der abgebildeten Entwicklungsreihe wurden die vorhergehenden $Z$ wischenstadien nicht beobachtet. Nach den Beobachtungen an anderen Eiern rundet sich der langgestreckte Zellhaufen allmählich ab; so entsteht die Sterroblastula. Eine Vorstufe des Sternchenstadiums ist in Abb. 3 dargestellt, an dem die ersten Anlagen der Tentakel sichtbar werden. 
Die Proactinula, das jüngste in den Planktonfängen gefundene Stadium, hat in der Aufsicht ein asteroiden-ähnliches Aussehen. Von der flachen zentralen rundlichen Scheibe sind durchschnittlich 10-13 Fortsätze, die Anlagen der aboralen Tentakel, deutlich abgesetzt (s. u. S. 244, Tab. 1). Bei einem etwas jüngeren Stadium als dem in Abb. 4 a, b dargestellten sind die Tentakel im Verhältnis zum Scheibendurchmesser noch etwas kürzer.

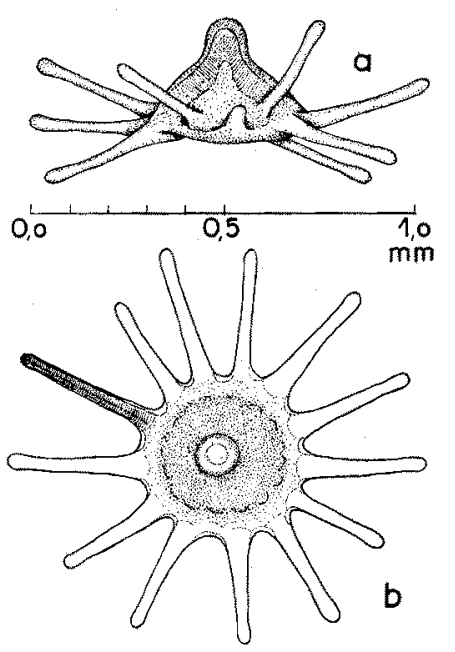

Abb. 4 a, b. Ectopleura dumortieri, "Sternchenstadium" = "Proactinula“ a) von der Seite, b) von oben. Abb. Zeichenapp. v. Leitz.

Die Seitenansicht zeigt, daß der auf der Unterseite flache Keim am oberen Pol eine leichte Aufwölbung besitzt, die bei der weiteren Entwicklung zum oralen Pol wird. Ektoderm und Entoderm sind bereits gesondert; ebenso ist auch schon die Gastralhöhle vorhanden, die wahrscheinlich durch Spaltbildung in der anfangs soliden Masse der großzelligen Entodermzellen entsteht. Die Tentakel, die solide sind und im Innern die geldrollenartig hintereinander angeordneten Entodermzellen enthalten, sind schwach geknöpft und tragen an der distalen Verdickung zahlreiche Nesselzellen. Für die Stellung der Tentakel ist typisch, daß sie nicht sämtlich in einer Ebene, sondern daß sie nach oben und unten alternierend getragen werden, wie in der Seitenansicht angedeutet ist.

Wesentlich für das Stadium der Proactinula sind daher: die Sternchenform, die kurze Längsachse, deren oberer Pol als Anlage des kegeligen Mundpoles schon vorhanden ist, ferner das Fehlen einer Differenzierung des aboralen Poles, der nur durch die Tentakelanlagen kenntlich ist. Diese sind in einer Zahl von 10-13 ausgebildet und dienen als Schwebefortsätze. Die ganze Formbildung der Proactinula weist darauf hin, daß es sich um ein planktisches Stadium handelt.

Die weitere Entwicklung zum Stadium 2 der eigentlichen Actinula ist einmal durch die Verlängerung der Tentakel gekennzeichnet, dann vor allem durch die Verlängerung der Körperachse. So wird die anfangs flache, dann breit konische Scheibe zum definitiven Actinula-Körper umgeformt. Bei der Differenzierung des Mundpoles, an dem der Durchbruch des Mundes erfolgt, wachsen $4-5$ kurze orale Tentakel aus. Ein weiteres wesentliches Merkmal ist 
die Ausformung des aboralen Poles zu der Anlage des Hydrocaulus mit der unteren Haftplatte. Bei dem in Abb. 5 dargestellten Stadium, das 48 Std. nach der Eiablage erreicht war, wurden die aboralen Tentakel in eigenartiger Weise über dem aboralen Pol zusammengelegt getragen. Diese Haltung bewirkt zweifellos eine Erhöhung der Sinkgeschwindigkeit; daher wird vermutet, daß3 die ansatzreife Actinula eine ähnliche Haltung einnimmt, wenn sie sich zu Boden sinken läßt. Am 3. Tage konnte die erste Nahrungsauf nahme beobachtet werden, noch ehe sich die Actinula angeheftet hatte (s.u. S. 245). Sie hatte jetzt die in Abb. 6 dargestellte Form, die durch die Weiterentwicklung des aboralen

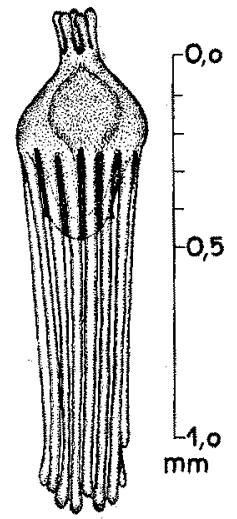

Abb, 5. Ectopleura dumortieri, Actinula, die ihre aboralen Tentakel über dem aboralen Pol zusammengelegt hat. Abb. Zeichenapp. v. Leitz.

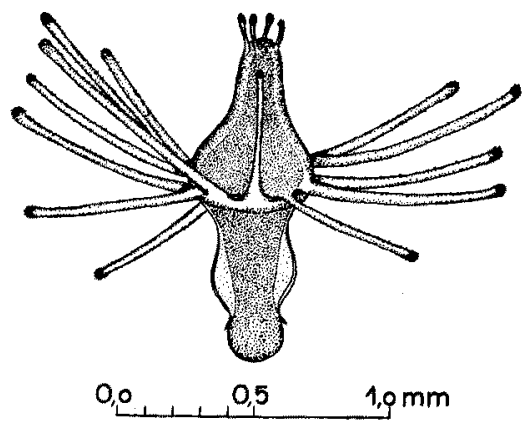

Abb. 6. Ectopleura dumortieri, Actinula mit deutlich entwickelter Anlage des Hydrocaulus am aboralen Pol. Abb. Zeichenapp. v. Leitz.

Poles zur Anlage des Stieles mit der Haftplatte gekennzeichnet ist und als Typus der Tubulariiden-Actinula gelten kann. Unterhalb des Ringwulstes, über dem die aboralen Tentakel sitzen, bildet sich auf der Außenseite der Hydrocaulus-Anlage bereits das feine und durchsichtige Periderm. Das ist bei einem etwas weiter entwickelten Stadium (Abb. 7) noch deutlicher, das unmittelbar vor der Festheftung steht.

Der früheste Zeitpunkt der Anheftung war der 4. bis 5. Tag nach der Eiablage. Bei zwei Actinulae, die sich in der gleichen Kulturschale befanden, konnte beobachtet werden, daß sich die eine am Boden des Gefäßes, die andere dagegen am Stiel der ersten anheftete (Abb. 8). Der am Glasboden angeheftete Polyp war offenbar zuerst zum Bodenleben übergegangen und gab 
so der zweiten Actinula eine Gelegenheit zur Anheftung, die sie dem glatten Glasboden vorzog. Dies entspricht wahrscheinlich dem Normalverhalten von Ectopleura, da die Polypen wie erwähnt oft an Stöckchen anderer Hydrozoen angeheftet gefunden werden. Zu einem späteren Zeitpunkt boten die beiden

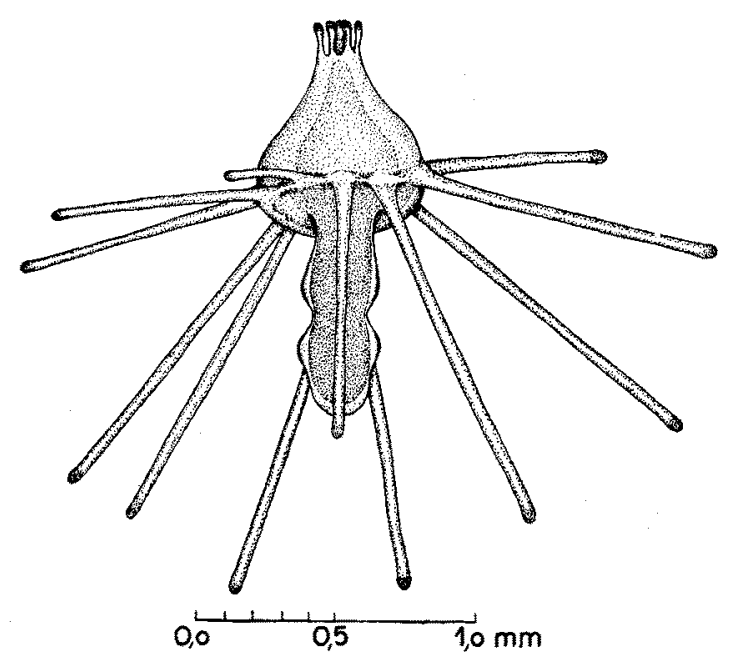

Abb. 7. Ectopleura dumortieri, Actinula im Stadium des Oberganges zum Bodenleben. Abb. Zeichenapp. v. Leitz.

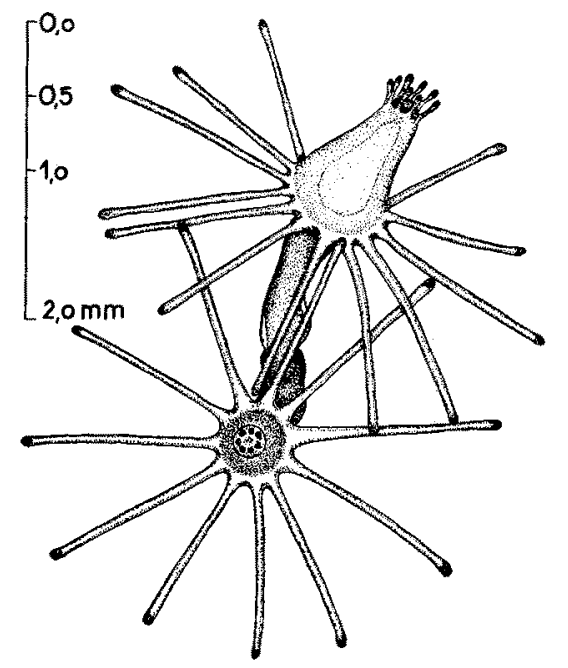

Abb. 8. Ectopleura dumortieri, zwei junge, gleichalte Polypen, von denen sich der obere (in Seitenansicht) am Stiel des unteren (in Aufsicht) angeheftet hat. Abb. Zeichenapp. v, Leitz.

Polypen das typische Bild einer Verzweigung, so daß man ohne Kenntnis des Sachverhaltes auf eine Verzweigung durch seitliche Sprossung hätte schließen müssen. Daher besteht die Möglichkeit, daß die für den Polypen von Ectopleura beschriebene Fähigkeit der seitlichen Verzweigung (MAYER 1910) in der gleichen Weise erklärt werden muß, daß also der Polyp durchaus solitär ist.

Die Weiterentwicklung der angehefteten Actinula zum Polypen erfolgt kontinuierlich; sie ist in erster Linie durch das starke Längenwachstum des 
Hydrocaulus gekennzeichnet; weiterhin durch die Größenzunahme des Polypenkopfes und die Vermehrung der oralen und aboralen Tentakel. Mehrfach wurde beobachtet, daß der ganze Polypenkopf nach Überfütterung abgeworfen wurde. Nach kurzer Zeit wurde er dann vollständig regeneriert, hatte dann aber zunächst eine geringere Größe und eine geringere Tentakelzahl. Wenige Tage nach der Anheftung traten dicht oberhalb des aboralen Tentakelkranzes die Anlagen der Medusenknospen in Erscheinung. Die Medusen kamen zur vollen Entwicklung und zur Ablösung von den schwach verzweigten, stielartigen Medusenträgern. Einzelne Polypen konnten monatelang am Leben gehalten werden. Daraus ist zu schließen, daß der Polyp die den Winter überdauernde Generation darstellt.

\section{Besprechung der Ergebnisse}

1. Nach den beschriebenen Beobachtungen ist die Entwicklung des Polypen von Ectopleura durch folgende Eigenschaften ausgezeichnet; a) die pelagischen Medusen erzeugen wenige große Eier, die sich nach dem Austritt aus der Gonade sofort vom Manubrium ablösen und pelagisch sind; b) sie entwickeln sich direkt unter Überschlagung der bewimperten Planula zu dem Stadium der wenig differenzierten, sternchenförmigen Proactinula, die ein echtes Planktonstadium darstellt; c) die Proactinula entwickelt sich zu dem planktischen. Stadium der eigentlichen, volldifferenzierten und ansatzreifen Actinula, die eine typische Tubulariiden-Actinula ist.

Die Entwicklungsdauer ist gering; sie betrug vom Ei bis zur ansatzreifen Actinula bei einer Zimmertemperatur von $17-19^{\circ}$ C 3-4 Tage. In der Zeit vom 4. zum 5. Tage erfolgte die Anheftung. Daher ist anżunehmen, daß auch die Entwicklung im freien Wasser nur wenige Tage in Anspruch nimmt, selbst wenn man berücksichtigt, daß bei den geringeren Temperaturen die Entwick. lung etwas langsamer ablaufen wird. Dabei ist allerdings zu berücksichtigen, daß die ansatzreife Actinula möglicherweise die planktische Phase über die normale Dauer hinaus verlängern kann, wenn nicht sofort ein geeigneter Ansiedlungsort gefunden wird. Diese Fähigkeit ist von metamorphosereifen Larven vieler anderer Bodentiere bekannt (Thorson 1946, 1950, 1952). In der Familie Tubulariidae ist allem Anschein nach die planktische Entwicklung vom Ei bis zur Actinula auf Ectopleura beschränkt; insbesondere ist die sternchenförmige planktische Proactinula nur dieser Gattung zuzuschreiben.

2. Bei Ectopleura durchläuft nämlich das Ei planktisch prinzipiell ähnliche Stufen der Entwicklung, wie sie bei Tubularia in das sessile Gonophor verlagert sind und bei Hybocodon am Manubrium der Meduse ablaufen. Die Ahnlichkeit in der Entwicklung ist vor allem bei Tubularia weitgehend, da bei dieser Gattung im Anschluß an die Sonderung der Keimblätter und im Zusammenhang mit der beginnenden Formbildung der Actinula ein sternförmiges Stadium auftritt, das mit einer frühen Ausbildungsstufe der oben beschriebenen Proactinula von Ectopleura in der Form und wohl auch im inneren Aufbau übereinstimmt. BRINK (1925) hat dieses sternförmige Stadium für Tubularia larynx beschrieben und darauf hingewiesen, daß es aus der älteren Literatur auch für $\mathcal{T}$. indivisa und $\mathcal{T}$. mesembryanthemum bekannt ist (vgl. p. 61, 63, Textfig. H 1, I 1 und Taf. II, fig. 1 a). Die Sternform ist wie bei Ectopleura durch die zuerst entstehenden Anlagen der aboralen Tentakel be- 
dingt. BRINK hat sogar beobachtet, daß ein solches sternförmiges Stadium von Tubularia larynx aus dem Gonophor geschlüpft war und sich auf dem Stiel eines älteren Polypen festgeheftet hatte. Dieses Verhalten ist aber zweifellos eine Ausnahmeerscheinung, da normalerweise die Entwicklung des sternförmigen Stadiums bis zur schlüpfreifen Actinula innerhalb des Gonophors stattfindet, wie durch eigene Beobachtungen an $\mathcal{T}$. larynx bestätigt werden kann. Daher ist ein sternförmiges Entwicklungsstadium von Tubularia im Plankton nicht zu erwarten.

Die Frage, ob in der Entwicklung der Actinula von Hybocodon ein sternförmiges Stadium auftritt, kann direkt nicht beantwortet werden, da die bisherigen Beschreibungen nicht sehr eingehend sind. Nach der Bemerkung von PERkins (1904, p. $516 \mathrm{f}$.) "Ten tentacles appear while the larva is still a spherical mass ..." ist diese Frage aber wohl zu verneinen. Aus der Abbildung eines Längschnittes durch eine am Manubrium der Meduse angeheftete Actinula bei HargitT (1917, Pl. 4, fig. 39) ist weiterhin zu entnehmen, daß der Körper der Actinula bereits vor der Loslösung eine ziemlich langgestreckte Form hat, und daß insbesondere die Region des aboralen Poles unterhalb der aboralen Tentakel schon relativ stark ausgebildet ist. Daher läßt sich wohl auch für Hybocodon das Vorkommen eines sternförmigen Stadiums der Proactinula im Plankton mit großer Sicherheit ausschließen. In jedem Fall ist wie bei Tubularia auch bei Hybocodon die schlüpfreife bzw. ablösungsreife Actinula das Endglied der larvalen Entwicklung. Die Actinulae beider Gattungen kommen daher im freien Wasser vor, und es ist zu prüfen, ob sie von der Actinula von Ectopleura unterschieden werden können.

3a) Die frischgeschlüpfte Actinula von Tubularia larynx, der häufigsten Art, die hier allein zum Vergleich herangezogen werden kann, befindet sich nach den Abbildungen und Angaben von Pyefinch u. Downing (1949) auf einem ähnlichen Entwicklungsstand wie das entsprechende Stadium von Ectopleura; doch lassen sich allem Anschein nach morphologische Unterscheidungsmerkmale finden. Der Körper der Actinula von $\mathcal{T}$. larynx wird von diesen Autoren als „ovoid“ (p. 24) oder "spherical“ (p. 41) bezeichnet. Er ist daher im ganzen plumper als der der Actinula von Ectopleura. Das gilt vor allem für den aboralen Pol mit der Anlage des Hydrocaulus und des Haftorgans, die bei Tubularia eine wesentlich breitere und massigere Form hat, wie eigene Kontrollbeobachtungen bestätigten. Die Länge des Körpers, gemessen vom aboralen bis zum oralen $\mathrm{Pol}$ einschließlich der oralen Tentakel, scheint bei beiden Arten nicht wesentlich verschieden zu sein. Dagegen unterscheiden sie sich deutlich in der Zahl der aboralen Tentakel. Sie betrug bei den nicht sehr zahlreichen Actinulae von Ectopleura, die aus Eiern gezüchtet waren, 10-13. Diese Zahlen stimmen mit den vorläufigen Ergebnissen von Zählungen überein, die AUrich an seinem Material aus Planktonfängen der südlichen Nordsee durchführte und die er mir freundlicherweise zur Verfügung stellte (Tab. 1).

Wenn auch die Gesamtzahlen für die untersuchten Stadien nicht sehr groß sind, so zeigen sie doch, daß sowohl bei der Proactinula wie bei der Actinula das Häufigkeitsmaximum für die aboralen Tentakel bei 12 liegt, während nach Pyerinch u. Downing die Zahl der aboralen Tentakel bei der Actinula von T. larynx durchschnittlich 9-11 beträgt. Eigene Beobachtungen führten zu dem gleichen Ergebnis.

Auch die Länge der aboralen Tentakel dürfte ein Unterscheidungsmerkmal sein, da sie bei T. larynx im Verhältnis zum Körper wesentlich länger 
sind als bei Ectopleura. Für Tubularia ist auch noch besonders die sehr starre Form der Tentakel charakteristisch, die bei der frischgeschlüpften Actinula in einer auffallend alternierend-gespreizten Form getragen werden, wie es mir in diesem Maße bei der Actinula von Ectopleura nicht aufgefallen ist. Bei Tubularia werden die aboralen Tentakel nach dem Festheften relativ kürzer und erst jetzt kontraktil, was in ähnlicher Weise auch bei Ectopleura zu beobachten ist.

Tabelle 1

Zahl der Oral- und Aboraltentakel bei der Proactinula und Actinula von Ectopleura dumortieri, nach Zählungen von AURICH an fixierten Stadien aus dem Plankton der südlichen Nordsee.

Stadium 1. Proactinula

\begin{tabular}{lrrrrrrrrrc}
\hline Zahl d. Aboraltentakel & 9 & 10 & 11 & 12 & 13 & 14 & 15 & 16 & 17 & Gesamtzahl \\
Zahl d. Tiere & 1 & 2 & - & 9 & 4 & 2 & 2 & 1 & 1 & 22
\end{tabular}

Stadium 2, Actinula

\begin{tabular}{llllllllllrrrrc}
\hline Zahl d. Oral-, & 4 & 5 & 6 & 7 & 8 & & & & & & & \\
d. Aboraltentakel & & & & & & 8 & 9 & 10 & 11 & 12 & 13 & 14 & Gesamtzahl \\
Zahl d. Tiere & 3 & 8 & 4 & 1 & 3 & 1 & - & 2 & 4 & 9 & 1 & 2 & 19
\end{tabular}

In der Form und Zahl der oralen Tentakel sind die Unterschiede weniger deutlich. Die Zahl beträgt nach meinen Beobachtungen bei beiden Arten 4-5, was mit den Angaben von Pyefinch u. Downing in Übereinstimmung steht. Nach den Zählungen von Aurich kann die Actinula von Ectopleura bis zu 8 Oraltentakel haben.

BRINK (1925, p. 71 f.) hat noch darauf hingewiesen, daß die Oraltentakel bei Tubularia nach den Angaben einiger Autoren bereits vor dem Schlüpfen ausgebildet werden, nach anderen aber erst nach dem Schlüpfen. Nach LowE (1924) und Pyefinch u. Downing (1949) besitzt die Actinula von T. larynx die Oraltentakel bereits im Gonophor, was durch eigene Beobachtungen bestätigt werden kann.

Nach allem dürfte es möglich sein, die schlüpfreife bzw. frischgeschlüpfte Larve von $\mathcal{T}$. larynx von der ansatzreifen Actinula von Ectopleura zu unterscheiden. Die Wahrscheinlichkeit, beide Formen im Plankton gleichzeitig anzutreffen, ist aber wohl gering, da Tubularia eine ausgesprochene Litoralform ist, deren Actinulae sich nach dem Ausschlüpfen sehr schnell festheften, während die Actinulae von Ectopleura durch ihre pelagische Entwicklung auch dem Hochseeplankton angehören können (s. u. S. 245).

b) Wie bereits erwähnt, ist die Entwicklung der Actinula von Hybocodon nicht sehr eingehend beschrieben. Bei dieser entwickeln sich zuerst die aboralen, später an dem dem Manubrium zugewandten Pol die oralen Tentakel und der Mund. Nach BRowne (1895) lösen sich die Actinulae zuweilen vom Manubrium der Meduse los, ehe die Oraltentakel und die Mundöffnung gebildet werden, was von Perkins (1904) als Regel angegeben wird. Die Zahl der aboralen Tentakel beträgt nach BROWNE $11-17$, die der oralen 8 . Nach diesen spärlichen Angaben läßt sich nicht ermitteln, ob die Actinula von Hybocodon nach ihrer Loslösung vom Manubrium der Meduse von der ansatzreifen Actinula von Ectopleura unterschieden werden kann. Auch über die Dauer der planktischen Phase liegen für Hybocodon keine Angaben vor. Nach Browne (1905) kommen die Actinulae von $H$. prolifer im Plankton zeitweilig häufig vor. Bei 
Plymouth tritt die Meduse von Hybocodon in der Zeit vom Februar bis zum Juni auf, mit dem Höhepunkt der Häufigkeit im Mai, während das Vorkommen von Ectopleura von April bis Mai und vom September bis November angegeben wird (Russele 1953) ${ }^{1}$ ). Daher ist die Möglichkeit nicht auszuschließen, daß die Actinulae beider Arten im Plankton gleichzeitig vorkommen. Eine eingehendere Beschreibung der morphologischen Merkmale des Actinulastadiums von Hybocodon und seiner Entwicklung ist aus diesem Grunde sehr erwünscht.

4. Bei einer vergleichenden Betrachtung der Entwicklung von Ectopleura, sowie von Tubularia, Hybocodon und Margelopsis lassen sich Gesichtspunkte finden, die sich auf die Ausprägung der planktischen Periode des Polypen beziehen.

Die Actinula von Tubularia kommt, wie bereits erwähnt, kurze Zeit nach dem Schlüpfen aus dem sessilen Gonophor im freien Wasser vor, ist aber allem Anschein nach nicht als echtes Planktonstadium anzusprechen, da sie sich unmittelbar nach dem Schlüpfen und meist in geringer Entfernung vom Ausgangspolypen festsetzt. Tubularia ist in ihrem ganzen Lebenscyclus eine reine Litoralform, so daß dementsprechend ihre Actinulae für die kurze Zeitspanne zwischen Ausschlüpfen und Festheften auf das Litoralplankton beschränkt sein müssen.

Wie weit die Actinula von Hybocodon nach der Ablösung vom Manubrium der Meduse als echtes Planktonstadium angesehen werden kann, läßt sich direkt nicht mit Sicherheit entscheiden, weil zu wenig über ihre Entwicklung bekannt ist. Nach der oben mitgeteilten Angabe (s. S. 244) über das zeitweilig häufige Vorkommen im Plankton ist aber damit zu rechnen. Diese indirekte Schlußfolgerung gewinnt an Wahrscheinlichkeit, wenn man berücksichtigt, daß die freien Medusen von Hybocodon, die wohl auch in Küstennähe erzeugt werden, auf die Hochsee hinausgetragen werden, wo sich daher auch die Actinulae von ihnen ablösen. Gegenüber Tubularia haben die Actinulae von Hybocodon also sehr wahrscheinlich eine echte, wenn auch vermutlich nicht sehr lange planktische Periode.

Das Gleiche gilt mit Sicherheit für die Actinulae von Ectopleura. Darüber hinaus konnte gezeigt werden, daß bei dieser Form die gesamte Entwicklung vom $\mathrm{Ei}$ bis zur ansatzreifen Actinula rein planktisch erfolgt. Daß mithin die Actinula vorübergehend ein echtes Planktonstadium ist, geht auch daraus hervor, daß sie schon vor dem Obergang zum Bodenleben Nahrung aufnehmen kann. Das konnte bei den aus Eiern gezüchteten Stadien direkt beobachtet werden (s. o. S. 240) und wurde mir von Aurich nach seinen Untersuchungen am Planktonmaterial bestätigt. Er fand nämlich nicht selten Larven, die Nahrungstiere im Magen hatten, so vor allem Copepoden; sie waren mehrfach so groß, daß der Körper der Actinula sich stark gedehnt und eine verzerrte Form angenommen hatte. Bei Ectopleura ist nach allem in den Lebenscyclus des Polypen eine verlängerte Periode des echten planktischen Daseins eingeschaltet.

Das legt einen Vergleich mit Margelopsis nahe, bei der der Polyp ständig pelagisch lebt. Die sich aus den Subitaneiern am Manubrium der Meduse entwickelnden Actinulae behalten nach ihrer Ablösung die planktische Lebens-

1) Herrn Prof. Russexl, Plymouth, möchte ich für Literaturhinweise auch an dieser Stelle herzlich danken. 
weise bei und entwickeln sich kontinuierlich zu den Polypen, die durch Knospung wieder die Medusen erzeugen. Margelopsis stellt daher den in Richtung auf die pelagische Lebensweise des Polypen am weitesten fortgeschrittenen Typ dar. Doch ist der Polyp auch bei dieser Form nicht rein pelagisch, da im Lebenscyclus ein langdauerndes embryonales Bodenstadium vorkommt (WERNER 1954, 1955 a, b). Die größeren Dauereier entwickeln sich am Manubrium der Meduse nur bis zur Sterroblastula, die sich ablöst, zu Boden sinkt und sich hier anheftet. Die Weiterentwicklung erfolgt nach einer mehrmonatigen winterlichen Ruheperiode, so daß erst im Frühjahr ein kleiner Polyp ausschlüpft, der zum Planktonleben übergeht.

Eine ähnliche Entwicklungsweise über ein am Boden angeheftetes Embryonalstadium findet sich (außer bei dem Süßwasserpolypen Hydra und ferner bei der Süßwassermeduse Limnocnida indica $\mathrm{n}$. RaO 1931) nur noch bei Corymorpha (s. o. S. 236), mit dem Unterschied, daß der Polyp bei C. nutans bereits nach $6-7$ Tagen aus dem $\mathrm{Ei}$ ausschlüpft und sofort zum Bodenleben übergeht; eine längere Ruheperiode fehlt hier also. Mit dem doppelten Entwicklungsmodus vereinigt daher Margelopsis die Fortpflanzungsweisen von Hybocodon und Corymorpha. Mit der letzteren Gattung hat Margelopsis überdies das Fehlen eines echten Periderms gemeinsam, da die einzige peridermale Bildung im Lebenscyclus die Schutzhülle des Bodendauerstadiums ist. Berücksichtigt man bei Corymorpha noch die Fähigkeit der langsamen Ortsänderung beim jungen wie erwachsenen Polypen, so ist man versucht, Corymorpha an den Anfang einer Reihe zu stellen, die über Tubularia, Hybocodon, Ectopleura zu Margelopsis führt und auf eine immer stärkere Ausprägung und Verlängerung der beweglichen bzw. planktischen Phase des Polypen gekennzeichnet ist. Auf diesen Zusammenhang wurde bereits früher kurz hingewiesen (WERNER 1954, $1955 \mathrm{a}$ ).

Indessen ergibt sich bei weiterer Prüfung des Sachverhaltes, insbesondere bei Einbeziehung des phylogenetischen Gesichtspunktes ein anderes Bild, so daß die obige Reihe, die sich auf die äußeren Merkmale der Entwicklung und die Beweglichkeit des Polypen bzw. die Dauer seiner planktischen Phase gründet, nicht als phylogenetische Entwicklungsreihe zu deuten ist. Die Trennung der Tubularia-Linie von der Corymorpha-Linie durch Kramp (s.o. S. $235 \mathrm{f}$.) erscheint wohl begründet; daher sind die aufgezeigten Ähnlichkeiten in der Entwicklung und im Verhalten nur als Konvergenzen zu werten (vgl. Kramp 1949, p. 207 f.). Andererseits lassen sich aber aus der Entwicklungsgeschichte innerhalb der Tubularia-Linie Hinweise für die phylogenetische Entwicklung ableiten.

Die rein planktische Entwicklung von Eclopleura beansprucht nämlich ein besonderes Interesse aus dem weiteren Grunde, weil sie die Bedeutung des Larvenstadiums der Actinula in einem neuen Licht erscheinen läßt. KüHN (1913, p. 45 f.) sieht in den Actinulae, die bei systematisch ganz verschiedenen Gruppen auftreten, kein "phyletisches Larvenstadium", sondern "Jugendstadien des speziellen Polypen", was zweifellos zutrifft. Sie sind dadurch charakterisiert, daß „... zwischen der Organisation der Larve und der des fertigen Polypen kein scharfer Gegensatz ..." besteht. „Den Augenblick der Festheftung an der Unterlage kann man als Grenze des Larvenlebens ansprechen, im übrigen aber ist der Übergang in das Polypenstadium eine ganz allmähliche Anderung, wie sie stets auch mit der gewöhnlichen Ausbildung des Polypen von einer Planula aus verbunden ist" (p. 46). 
Die gleichmäßige und kontinuierliche Entwicklung ist am deutlichsten bei Margelopsis infolge der planktischen Lebensweise des Polypen ausgeprägt, so daß hier die Actinula kein eigentliches Larvenstadium mehr darstellt. Der erwachsene Polyp unterscheidet sich von der eben losgelösten Actinula nur durch die Größe, die vermehrte Tentakelzahl und die Medusenknospung, während bei allen anderen Formen der Übergang zum Bodenleben immerhin eine tiefergreifende Umformung bedeutet, die morphologisch durch das Auswachsen des Hydrocaulus gekennzeichnet ist.

Zur Entstehung der Actinulae bemerkt nun KüHN (1. c., p. 46): „Ihr Dasein als freie Larven verdanken sie lediglich einer von den einzelnen Arten erst in späten Stadien der Stammesgeschichte als besondere Anpassung erworbenen Verlängerung des Lebens im Schutze des Mutterorganismus“. Diese Deutung erscheint begründet für Tubularia, Hybocodon und Margelopsis, hat aber keine Gültigkeit für Ectopleura, da ja bei dieser Form dàs zweifellos als ursprünglich zu bezeichnende Verhalten der Loslösung der Eier vom Manubrium und ihrer planktischen Entwicklung erhalten geblieben ist. Gleichwohl fehlt auch hier die Planula, und es erfolgt die direkte Entwicklung zur Actinula. Eine Erklärung für die Entstehung der Actinula erscheint von hier aus schwierig.

Die stammesgeschichtlich ursprünglichere und allgemeinere Erscheinung ist dem Anschein nach eben die Unterdrückung der Planula, die vielleicht mit der Größe und dem Dotterreichtum und mit der Zahl der Eier zu erklären ist. Es ist bekannt, daß bei Hybocodon, Ectopleura, Tubularia, Margelopsis u. a. die Eireifung in der besonderen Form erfolgt, daß die zur definitiven Eizelle heranreifende Oocyte das Material von zahlreichen sich auflösenden Oogonien oder die ganzen Oogonien in sich aufnimmt. Das hat zur Folge, daß meist nur wenige, aber um so größere Eier gebildet werden, worauf schon Müller (1908) hingewiesen hat. Die direkte Entwicklung ohne Planula, die mit Ausnahme von Ectopleura überdies im Schutze des mütterlichen Organismus erfolgt, hat dann die Bedeutung der Abkürzung der planktischen Phase als des größten Gefahrenmoments im Leben des Organismus und besonders der jüngsten Stadien. Der daraus entstehende Nachteil der geringeren Verbreitungsmöglichkeit wird durch die wenn auch wahrscheinlich allgemein nur kurze pelagische Periode des jugendlichen Polypen, eben der Actinula, mehr oder weniger ausgeglichen. Das dürfte bei der geringen Eiproduktion für die Erhaltung der Art von besonderer Bedeutung sein.

Da nach KüHN (1913, p. 215) bei Formen mit freien Medusen durchweg zahlreiche Eier gebildet werden, in den sessilen Gonophoren aber nur wenige, so kann man von Ectopleura und Hybocodon, die ja trotz freier Gonophoren nur wenige Eier bilden, vermuten, daß sie sich eben auf dem Wege zur Reduktion der Medusen befinden, wie es bei Tubularia bereits erreicht ist. Dieselbe Vermutung ist dann auch für Corymorpha-Steenstrupia auszusprechen, wo die gleiche Erscheinung der geringen Eiproduktion bei freien Medusen zu beobachten ist. Dafür spricht ebenfalls, daß bei $C$. palma die Meduse sich nicht mehr loslöst; sie besitzt zwar noch einen wohl ausgebildeten Schirm, der aber verkleinert ist und der Tentakel entbehrt (ToRREY 1907). Bei C. nutans ist dagegen die Meduse noch pelagisch.

Aus der Entwicklungsgeschichte ist nach allem zu folgern, daß in der $\mathrm{Fa}-$ milie Tubulariidae die Gattung Ectopleura die ursprünglichere vor Hybocodon und Tubularia ist. Die bisher nach morphologischen Gesichtspunkten durchgeführte Einordnung wird damit bestätigt.

Der Übergang zur pelagischen Lebensweise beim Polypen von Margelopsis muß dann als spezialisierte Weiterentwicklung in anderer Richtung be- 
trachtet werden. Das Vorkommen eines embryonalen Bodenstadiums zeigt, daß die Polypengeneration nicht rein pelagisch ist. Das läßt darauf schließen, daß Margelopsis von Vorfahren mit festsitzenden Polypen abstammt, daß also die planktische Lebensweise des Polypen eine sekundär erworbene Eigenschaft darstellt. In die gleiche Richtung deutet auch das Vorhandensein des Rudiments der Stielanlage am aboralen Pol des Polypen, das der Haftplatte der Tubulariiden-Actinula homolog ist, wie schon KüHN (1913) angegeben hat. Nach den eigenen Beobachtungen besteht diese Homologie nicht nur der Anlage, sondern auch der Funktion nach. Die Zellen dieser haftnapfartigen Bildung am aboralen Pol können nämlich ein klebriges Sekret ausscheiden. mit dem sich der in den Kulturen auf dem Boden liegende Polyp zeitweilig anheften kann (WERNER 1955 a).

Daher ist der Auffassung von Kramp (1949) zuzustimmen, daß sich Margelopsis von gemeinsamen Vorfahren frühzeitig abgespalten hat. Die phylogenetische Entwicklungslinie läßt sich in dem folgenden Zweigschema darstellen:

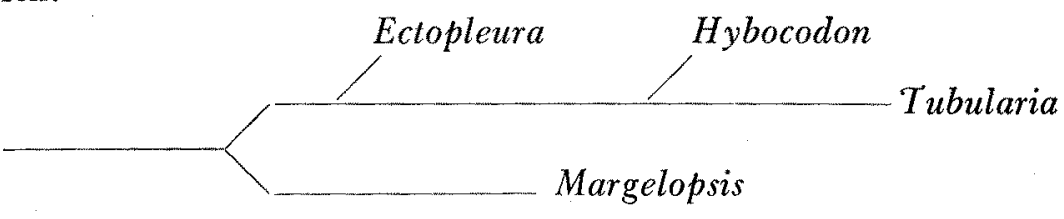

D. Zusammenfassung

1. Frisch gefangene Medusen von Ectopleura dumortieri van Beneden legten befruchtete Eier ab, so daß ihre Entwicklung untersucht werden konnte.

2. Die Eier lösen sich nach dem Austritt aus der Gonade vom Manubrium $\mathrm{ab}$ und entwickeln sich planktisch unter Überschlagung des bewimperten Planulastadiums direkt zur Actinula.

3. Das jüngste Actinula-Stadium ist das „Sternchenstadium“, die "Proactinula". Sie ist eine flache rundliche Scheibe mit 10-13 Fortsätzen, den Anlagen der aboralen Tentakel. Der Mundpol kann durch eine schwache Aufwölbung schon angedeutet sein, während der aborale Pol außer den Tentakeln noch keine Differenzierung aufweist. Die Proactinula ist nach ihrer Entwicklung und Formbildung ein planktisches Ausbreitungsstadium.

4. Die Proactinula entwickelt sich zu der eigentlichen planktischen Actinula. Sie ist nach ihrer Formbildung eine typische Tubulariiden-Actinula mit 10-13 langen aboralen Tentakeln und 4-5 kurzen Oraltentakeln am Mundpol. Am aboralen Pol ist die Anlage des Hydrocaulus mit der Haftplatte ausgebildet. Die Actinula vermag bereits vor der Anheftung Nahrung aufzunehmen.

5. Die Anheftung erfolgte am 4.-5. Tage nach der Eiablage. Die sich entwickelnden Polypen konnten mehrere Monate am Leben gehalten werden und bildeten an schwach verzweigten, stielartigen Medusenträgern die Medusen.

6. Die vollständige planktische Entwicklung vom $\mathrm{Ei}$ bis zur ansatzreifen Actinula ist in der Familie Tubulariidae allem Anschein nach auf Ectopleura beschränkt.

7. Die planktische Proactinula ist gleichfalls auf Ectopleura beschränkt, 
während im freien Wasser noch Actinulae von Tubularia und Hybocodon auftreten können. Die morphologische Unterscheidung der Actinulae von Ectopleura und Tubularia erscheint möglich. Doch ist unwahrscheinlich, daß die Actinulae beider Gattungen gleichzeitig im Hochseeplankton auftreten, da Tubularia eine ausgesprochene Litoralform ist.

8. Ebenso wie von Ectopleura können auch die Actinulae von Hybocodon im Hochseeplankton auftreten. $\mathrm{Ob}$ die morphologische Unterscheidung der Actinulae möglich ist, kann nicht entschieden werden, da über die Entwicklung des Polypen von Hybocodon zu wenig bekannt ist.

9. Durch die Beibehaltung der als ursprünglich zu bezeichnenden planktischen Entwicklung ist Ectopleura in der Familie Tubulariidae vor Hybocodon und Tubularia als die phylogenetisch ursprünglichere Gattung einzuordnen.

\section{E. Literaturverzeichnis}

Brink, R., 1925: Beiträge zur Herstellung einer rationellen Hydroidensystematik. Onderzoek. Zool. Laborat. Rijksuniv. Groningen, 8.

B r o ch, H., 1924: Hydroida. Kükenthal-Krumbach, Hdbch. d. Zool. 1.

- 1928: Hydrozoa I. Grimpe-Wagler, Tierwelt d. Nord- u. Ostsee, III b.

B rowne, E. T., 1895: Report on the medusae of the L.M.B.C. District. Trans. Liverpool Biol. Soc. 9.

- 1905: A report on the medusae found in the Firth of Clyde (1901-1902). Proc. Roy. Soc. Edinb. 25.

Hargitt, G. T., 1917: Germ cells of Coelenterates. III. Aglantha digitalis. IV. Hybocodon prolifer. J. Morph. 28.

Hartla ub, C., 1897: Die Hydromedusen Helgolands. Wiss. Meeresunters. N. F., 2.

- 1907: Craspedote Medusen. I, 1. Codoniden und Cladonemiden. Nord. Plankton, 12.

Korschelt, E. und Heider, K., 1936: Vergleichende Entwicklungsgeschichte der Tiere. Jena.

Kramp, P. L., 1930: Hydromedusae collected in the south-western part of the North Sea and in the eastern part of the Channel in 1903-1914. Mém. Mus. Roy. Hist. Nat. Belg. 45

- 1949: Origin of the hydroid family Corymorphidae. Vidensk. Medd. Dansk. naturh. Foren. 111.

Kühn, A., 1913: Entwicklungsgeschichte und Verwandschaftsbeziehungen der Hydrozoen. I. Dic Hydroiden. Ergebn. u. Fortschr. d. Zool. 4.

Künne, C., 1952: Untersuchungen über das Großplankton in der Deutschen Bucht und im Nordsylter Wattenmeer. Helgol. Wiss. Meeresunters. 4.

Lelou p, E., 1929: A propos de l'hydraire Margelopsis haeckeli Hartlaub. Ann. Soc. Roy. Zool. Belg. 60 .

Linko, A., 1904: Zoologische Studien im Barents-Meere. Zool. Anz. 28.

Lowe, E., 1926: The embryology of Tubularia larynx (Allm.). Quart. J. Micr. Sci. 70.

Mayer, A., 1910: Medusae of the world. Publ. Carnegie Inst. Wash. 109.

Müller, H., 1908: Untersuchungen über Eibildung bei Cladonemiden und Codoniden. $Z$. w. Z. 89.

Perkins, H. F., 1904: Double reproduction in the medusa Hybocodon prolifer. American Naturalist 38.

Pyef inch, K. A. und Downing, F. S., 1949: Notes on the general biology of Tutularia larynx Ellis u. Solander. J. Mar. Biol. Ass. 28.

R a o, H. S., 1931: The supposed resting stage of Limnocnida indica Annandale. Nature, 127.

Rees, W. J., 1937: The development of the hydroid Corymorpha nutans M. Sars from the egg. J. Mar. Biol. Ass. 21.

- 1941: Notes on British and Norwegian hydroids and medusae. Ibid. 25.

Russe1, F. S., 1938: The Plymouth off-shore medusa fauna. Ibid. 22.

- 1953: The medusae of the British Isles. Cambridge.

Stechow, E., 1923: Zur Kenntnis der Hydroidenfauna des Mittelmeeres, Amerikas und anderer Gebiete. Zool. Jahrb. System. 47. 
Torrey, H, B., 1907: Biological studies on Corymorpha II. The development of C. palma from the egg. Univ. Calif. Publ. Zool. 3.

Thorson, G., 1946: Reproduction and larval development of Danish marine bottom invertebrates, with special reference to the planctonic larvae in the Sound (Øresund). Medd. Komm. Danm. Fisk. og. Havunders., Ser. Plankton, 4.

1950: Reproductive and larval ecology of marine bottom invertebrates. Biol. Rev. 25.

- 1952: Zur jetzigen Lage der marinen Bodentier-Okologie. Verh. Dtsch. Zool. Ges. 1951.

Werner, B., 1954: On the development and reproduction of the Anthomedusan, Margelopsis haeckeli Hartlaub. Trans. New York Acad. Sci, Ser. II, 16. (Abstract).

- $1955 \mathrm{a}$ : On the development and reproduction of the Anthomedusan, Margelopsis haeckeli Hartlaub. Ann. New York Acad. Sci. Im Druck.

- 1955 b: Uber die Fortpflanzung der Anthomeduse Margelopsis haeckeli Hartlaub durch Subitan- und Dauereier und die Abhängigkeit ihrer Bildung von äußeren Faktoren. Verh. Dtsch. Zool. Ges. 1954. Im Druck. 